Research Paper

\title{
Dickkopf-1 expression is associated with tumorigenity and Iymphatic metastasis in human hilar cholangiocarcinoma
}

\author{
Xiang-de Shi ${ }^{1, *}$, Xian-huan $\mathbf{Y u}^{1,{ }^{*}}$, Wen-rui Wu ${ }^{1, *}$, Xiao-lin $\mathrm{Xu}^{2}$, Jie-Yu Wang ${ }^{3}$, \\ Lei-bo Xu' ${ }^{1}$, Rui Zhang ${ }^{1,4}$, Chao Liu ${ }^{1}$ \\ ${ }^{1}$ Guangdong Provincial Key Laboratory of Malignant Tumor Epigenetics and Gene Regulation and Department of \\ Biliary-Pancreatic Surgery, Sun Yat-sen Memorial Hospital, Sun Yat-sen University, Guangzhou, 510120, China \\ ${ }^{2}$ Department of Ultrasound, Sun Yat-sen Memorial Hospital, Sun Yat-sen University, Guangzhou, 510120, China \\ ${ }^{3}$ Department of Hematology, Sun Yat-sen Memorial Hospital, Sun Yat-sen University, Guangzhou, 510120, China \\ ${ }^{4}$ Faculty of Medicine, Department of Gastroenterology and Hepatology, University Duisburg-Essen, Essen, 45147, Germany \\ *These authors contributed equally to this work \\ Correspondence to: Rui Zhang, email: 13828409500@126.com \\ Chao Liv, email: 252991332@qq.com
}

Keywords: DKK 1, HCCA, tumorigenesis, lymphatic metastasis

Received: February 15, $2016 \quad$ Accepted: August 24, 2016

Published: September 06, 2016

\section{ABSTRACT}

Dickkopf-1 (DKK1) is involved in tumorigenesis and the invasion of several tumors. However, its biological function in human hilar cholangiocarcinoma (HCCA) has not yet been documented. This study was designed to investigate the clinical significance and biological function of DKK1 in HCCA. The expression of DKK1 was investigated in thirty-seven human HCCA biopsy samples by immunohistochemistry. To further explore the biological effects of DKK1 in HCCA, transient and stable knockdown of DKK1 in two human HCCA cells (QBC939 and FRH0201) were established using small interfering or short hairpin RNA expression vector. In the present study, immunohistochemistry revealed that DKK1 was up-regulated in human HCCA tissues $(24 / 37,64.9 \%)$. High levels of DKK1 in human HCCA correlated with metastasis to the hilar lymph nodes $(P=0.038)$. Genetic depletion of DKK1 in HCCA cells resulted in significantly inhibited proliferation, colony formation and migration compared with controls. Most importantly, DKK1 down-regulation impaired tumor formation capacity of HCCA cells in vivo. Subsequent investigations revealed that $\beta$-catenin is an important target of DKK1 and DKK1 exerts its pro-invasion function at least in part through the $\beta$-catenin/ matrix metalloproteinase-7 (MMP-7) signaling pathway. Consistently, in human HCCA tissues, DKK1 level was positively correlated with $\beta$-catenin and MMP-7 expression, as well as tumor hilar lymphatic metastasis. Taken together, our findings indicate that DKK1 may be a crucial regulator in the tumorigenicity and invasion of human HCCA, DKK1 exerts its pro-invasion function at least in part through the $\beta$-catenin/ MMP-7 signaling pathway, suggesting DKK1 as a potential therapeutic target for HCCA.

\section{INTRODUCTION}

Hilar cholangiocarcinoma (HCCA) is the second most common primary liver cancer, which originates from the hilar biliary duct epithelium and is a highly invasive cancer accounting for 50 to $60 \%$ of cholangiocarcinoma $[1,2]$. Epidemiologic studies indicate an increasing incidence of HCCA, which has become one of the major malignancies threatening human health in recent years [3].
Radical surgical resection is the most effective therapy for the treatment of HCCA [4]. Due to frequent local recurrence of the tumor, the postoperative 5-year survival rate is $<30 \%$, even in patients who undergo radical surgery $[5,6]$. For inoperable patients, the median survival time is 6 to 12 months and the overall 5 -year survival rate is $<5 \%[7,8]$. Regional and para-aortic lymph nodes are frequently involved in HCCA, and lymph node metastasis (LNM) remains one of its most important prognostic 
markers. Thus, biomarkers that can predict the risk of recurrence and metastasis are urgently needed. It is necessary to identify novel gene targets that participate in tumor progression and to design appropriate treatment strategies for HCCA patients.

The Dickkopf (DKK) family consists of four members (DKK1, DKK2, DKK3 and DKK4). DKK1 is the most studied member of the DKK family. DKK1 was originally identified as a head inducer after its mRNA was injected into Xenopus embryos [9]. In the canonical Wnt/ $\beta$ catenin pathway, Wnt1 protein binds to the frizzled receptor (Fz) and the low-density lipoprotein receptor-related protein $[10,11]$. It has been reported that the expression of DKK1 were downregulated, resulted in $\beta$-catenin degradation and retardation of proliferation of tumor cells $[12,13]$. However, recent studies have reported that DKK1 is up-regulated in many tumors, including breast cancer, lung cancer, esophageal carcinoma and hepatocellular carcinoma (HCC) [14-18]. Moverover, DKK1 is involved in the invasion of non-small cell lung cancer, pancreatic cancer and HCC [19-21]. Shi et al. demonstrated that high expression of DKK1 is related to lymphatic metastasis and is indicative of a poor prognosis in intrahepatic cholangiocarcinoma (ICC) patients after surgery. Conversely, depletion of DKK1 using small interfering RNA results in a decrease in ICC cell migration and invasion [22]. Taken together, these findings suggest that DKK1 performs an oncogenic and invasion function. However, whether DKK1 acts as an oncogene in HCCA remains unclear. Furthermore, the precise mechanism of DKK1 involvement in HCCA tumorigenesis and invasion has not been determined. This study was designed to investigate the clinical significance and biological function of DKK1 in human HCCA.

\section{RESULTS}

\section{The expression of DKK1 was elevated in HCCA tissues and associated with hepatic hilar lymph nodes metastasis}

Positive staining of DKK1 protein by immunohistochemistry was observed in the cytoplasm of tumor cells (Figure 1). The expression of DKK1 was upregulated in HCCA tissues compared with corresponding peritumoral tissues (Supplementary Figure S1A-S1B). In general, high DKK1 expression $(++$ or +++$)$ was observed in 24 of 37 tumor samples (64.9\%; Figure 1C-1D), whereas low DKK1 expression (- or + ) was noted in 13 of 37 tumor samples (35.1\%; Figure 1A-1B).

As shown in Supplementary Table S1, the level of DKK1 expression in HCCA tissues was significantly correlated with metastasis to hepatic hilar lymph nodes $(\mathrm{P}=0.038)$ and tumor differentiation $(\mathrm{P}=0.039)$. No statistically significant differences were noted in gender $(\mathrm{P}=0.489)$, nerve infiltration $(\mathrm{P}=0.495)$, vascular invasion $(\mathrm{P}=0.091)$, TNM stage $(\mathrm{P}=0.691)$ or serum CA19-9 $(\mathrm{P}=0.383)$.

\section{Transfection of DKK1-shRNA repressed the proliferation and colony formation capacity of QBC939 and FRH0201 cells in vitro}

To investigate the effect of DKK1 on HCCA cells, we depleted its expression in QBC939 and FRH0201 cells by shRNA-DKK1 and established stable cell lines. As shown in Figure 2, the qRT-PCR and Western blot results revealed that DKK1 is effectively and functionally suppressed in QBC939 and FRH0201 cells compared to the scramble control (Figure 2A). The results of western blot were quantified by Image $\mathrm{J}$ (Supplementary Figure $\mathrm{S} 1 \mathrm{C}$ ). To further explore the biological role of reduced DKK1 in of QBC939 and FRH0201 cells, proliferation assay and colony formation assays were performed. CCK8 cell proliferation assay revealed that the decrease in DKK1 expression caused by DKK1-shRNA significantly inhibited the proliferation of QBC939 and FRH0201 cells (Figure 2B). A colony formation assay, based on crystal violet staining, revealed that fewer colonies were found in the DKK1-shRNA-treated QBC939 and FRH0201 cells compared to the control(Figure 2C). Similar results were observed in QBC939 and FRH0201 cells tranfected with small interfering (si) RNA (Supplementary Figure S2A-S2D).Collectively, these data indicate that downregulation of DKK1 inhibits the proliferation and colony formation capacity of QBC939 and FRH0201 cells.

\section{Transfection of DKK1-shRNA impaired the migration of QBC939 and FRH0201 cells in vitro}

To examine whether the targeted down-regulation of DKK1 in QBC939 and FRH0201 cells affects the migration of tumor cells, in vitro wound healing assays were performed. We found that cells in the DKK1-shRNA group exhibited decreased migration ability compared with the control (Figure 3A-3B). Similar results were observed in QBC939 and FRH0201 cells tranfected with siRNA (Supplementary Figure S2E). Thus, down-regulation of DKK1 dramatically diminishes the migration of QBC939 and FRH0201 cells in vitro.

\section{Transfection of DKK1-shRNA impaired tumor formation of QBC939 and FRH0201 cells in vivo}

Because our in vitro studies suggested that DKK1 plays a regulatory role in QBC939 and FRH0201 cell proliferation and migration, the biological significance of these results was further evaluated in an in vivo model of HCCA. To this end, both DKK1-shRNA tumor cells and control cells were implanted subcutaneously into nude mice, and the resulting tumor was measured. Following down-regulation of DKK1 expression, QBC939 and FRH0201 cells exhibited significantly diminished in vivo tumor formation compared with control cells (Figure 4A). DKK1 expression in subcutaneous tumors was analyzed 
by immunohistochemistry. DKK1 expression was positively correlated with subcutaneous tumor volume, the representative images are shown in Figure 4B. These data corroborate our in vitro observations and support the notion that DKK1 may play a vital role on proliferation and tumorigenicity of HCCA cells.

\section{Knockdown of DKK1 repressed $\beta$-catenin expression in QBC939 and FRH0201 cells}

We next investigated a potential mechanism for DKK1-mediated HCCA cell migration and invasion. DKK1 is a key regulator of the $\mathrm{Wnt} / \beta$ catenin signaling pathway. We therefore examined whether DKK1 influenced $\beta$-catenin expression in HCCA. First, we analyzed $\beta$-catenin expression using immunohistochemistry in tissue from all 37 cases of HCCA. Interestingly, DKK1 levels were positively correlated with $\beta$-catenin expression (Figure 1A-1D and Table 1; $\mathrm{P}=0.021$ ). Consistently, Western blotting indicated that DKK1-shRNA inhibited $\beta$-catenin expression in QBC939 and FRH0201 cells compared with control cells (Figure 4C). Most importantly, the expression of nuclear $\beta$-catenin was impaired by DKK1-shRNA in QBC939 and FRH0201 cells (Figure 4D). The results of western blot were quantified by Image J (Supplementary Figure S1D$\mathrm{S} 1 \mathrm{~F})$. These findings indicate that $\beta$-catenin may be an important downstream target of DKK1.
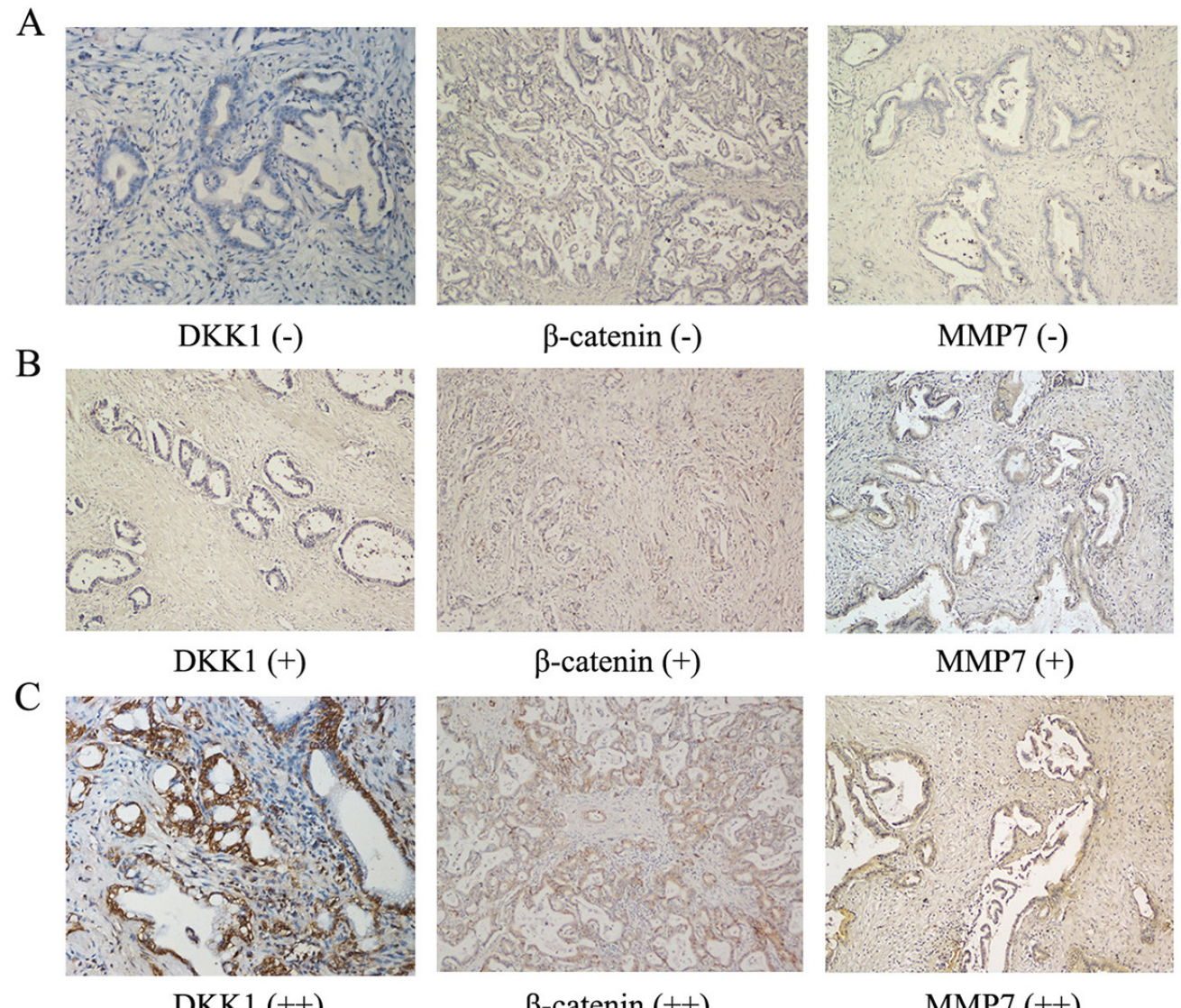

$\beta$-catenin (+)
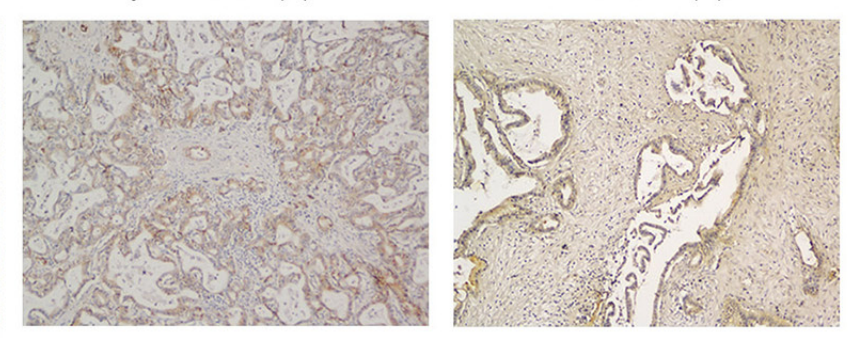

$\mathrm{D}$
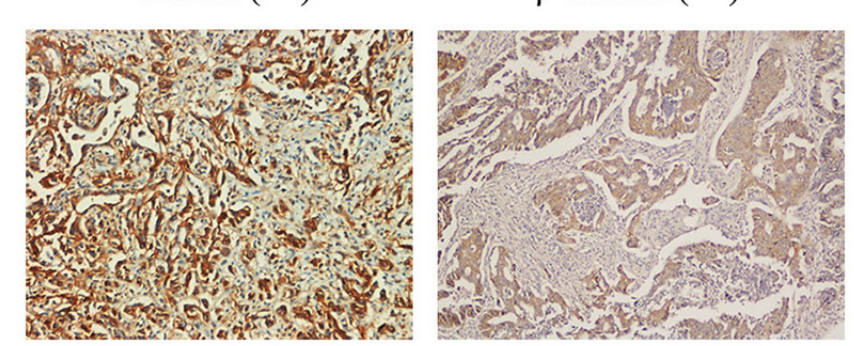

DKK1 (+++)

$\beta$-catenin (+++)

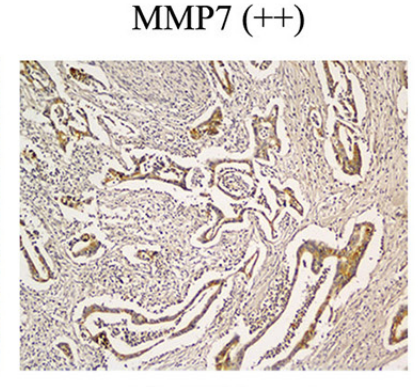

Figure 1: Expression of DKK1, $\beta$-catenin and MMP-7 in human HCCA tissues. Immunohistochemistry revealed no staining $(-)$ A. low staining $(+)$ B. moderate staining $(++)$ C. and strong staining $(+++)$ D. for DKK1, $\beta$-catenin and MMP-7 in human HCCA tissue. The DKK1 level was positively correlated with $\beta$-catenin and MMP-7 expression in human HCCA tissue (A-D). ${ }^{*} \mathrm{P}<0.05$. 
Table 1: Correlation between DKK1 expression and 37 patients with hilar cholangiocarcinoma

\begin{tabular}{|c|c|c|c|c|}
\hline \multirow[t]{2}{*}{ Variables } & & \multicolumn{2}{|c|}{ DKK1 } & \multirow[b]{2}{*}{$\mathbf{P}$} \\
\hline & & Positive & Negative & \\
\hline \multirow{2}{*}{ Sex } & Male & 15 & 6 & 0.489 \\
\hline & Female & 9 & 7 & \\
\hline \multirow{2}{*}{ CA19-9(U/L) } & $\geq 37$ & 18 & 12 & 0.383 \\
\hline & $<37$ & 6 & 1 & \\
\hline \multirow{2}{*}{ Tumor differentiation } & Well & 6 & 8 & 0.039 \\
\hline & Poor & 18 & 5 & \\
\hline \multirow{2}{*}{ Nerve infiltration } & Yes & 8 & 6 & 0.495 \\
\hline & No & 16 & 7 & \\
\hline \multirow{2}{*}{$\begin{array}{l}\text { Hilar lymphatic } \\
\text { metastasis }\end{array}$} & Yes & 15 & 3 & 0.038 \\
\hline & No & 9 & 10 & \\
\hline \multirow{2}{*}{ Vascular invasion } & Yes & 15 & 4 & 0.091 \\
\hline & No & 9 & 9 & \\
\hline \multirow{2}{*}{ TNM stage } & I-II & 5 & 4 & 0.691 \\
\hline & III-IV & 19 & 9 & \\
\hline \multirow{2}{*}{ Beta-catenin } & Positive & 16 & 4 & 0.021 \\
\hline & Negative & 7 & 10 & \\
\hline \multirow{2}{*}{ MMP-7 } & Positive & 18 & 5 & 0.039 \\
\hline & Negative & 6 & 8 & \\
\hline
\end{tabular}

\section{Matrix metalloproteinase-7 (MMP-7) was an important downstream target of the DKK1/ק-catenin signaling pathway}

MMP-7 is one of the most important target genes downstream of $\beta$-catenin signaling pathway and MMP-7 plays a crucial role in promoting cancer migration and invasion [23]. We therefore investigated whether DKK1 exerted its pro-invasion function through MMP-7 in HCCA. We analyzed MMP-7 expression using immunohistochemistry on tissues from all 37 cases of human HCCA. MMP-7 levels were positively correlated with DKK1 and $\beta$-catenin expression (Figure 1A-1D and Table 1; $\mathrm{P}=0.039$ ). Most importantly, the expression of DKK1, $\beta$-catenin and MMP-7 in HCCA were associated with hilar lymph nodes metastasis. Consistently, as shown in Figure $4 \mathrm{C}$, the introduction of shRNA-DKK1 remarkably decreased MMP-7 protein levels in QBC939 and FRH0201 cells. The results of western blot were quantified by Image J (Supplementary Figure S1D-S1F). Taken together, our findings suggest that DKK1 promotes HCCA cells migration and invasion, at least in part through promoting $\beta$-catenin/MMP-7 signaling.

\section{DISCUSSION}

DKK1 functions as key regulator of the canonical Wnt pathway [24]. DKK1 binds to LRP5/6 and dysregulation its interaction with Wnt, resulting in $\beta$-catenin degradation [10]. Numerous studies have demonstrated that DKK1 is involved in tumorigenesis in many types of cancer, including breast cancer, lung cancer, esophageal carcinomas, HCC and malignant bone disease $[15,25,26,27]$. However, the role of DKK1 in the progress of HCCA has not been documented. In the present study, we found that the expression of DKK1 is elevated in HCCA tissues, and the high level of DKK1 expression was correlated with hilar lymph node metastasis. These data indicate that DKK1 may involve in the lymph node metastasis of HCCA.

Studies have indicated that DKK1 is oncogenic and is involved in invasive growth in non-small cell lung cancer cells [19]. Chen et al. reported that DKK1 promotes the invasion and metastasis of HCC cells [21]. A recent research article demonstrated that high DKK1 expression is related to lymphatic metastasis and indicates poor prognosis in ICC [22]. However, the role of DKK1 in the invasion and tumorigenicity of HCCA cells is still unclear. Herein, our 
in vitro assays demonstrated that the depletion of DKK1 in these cells impaired proliferation, colony formation capacity and migration capacity. Most importantly, implantation of DKK1-shRNA HCCA cells into nude mice impaired their tumor formation capacity in vivo. Immuno-histochemistry revealed that DKK1 expression was positively correlated with subcutaneous tumor volume. These data corroborate our in vitro observations and support the notion that DKK1 may play a vital role on progression of HCCA.

We therefore studied the mechanism of DKK1 in the tumorigenesis and invasion of HCCA. DKK1 is a key regulator of $\mathrm{Wnt} / \beta$-catenin signaling pathway. The
Wnt/ $\beta$-catenin signaling pathway is highly conserved in evolutionary processes and is up-regulated in multiple tumors [28]. However, the role of DKK1 on the expression of $\beta$-catenin is controversial [29]. Although DKK1's ability to inhibit the canonical $\mathrm{Wnt} / \beta$-catenin signalling pathway have been identified first, it was later found that the DKK1 gene is also a target of $\mathrm{Wnt} / \beta$-catenin activation. It has been reported that DKK1 could down-regulate the expression of $\beta$-catenin in breast cancer, thyroid cancer and epidermal neoplasms [30-32]. In contrast, Chen et al. reported that up-regulation of DKK1 expression noticeably promoted the cytoplasmic and nuclear accumulation of $\beta$-catenin in
A
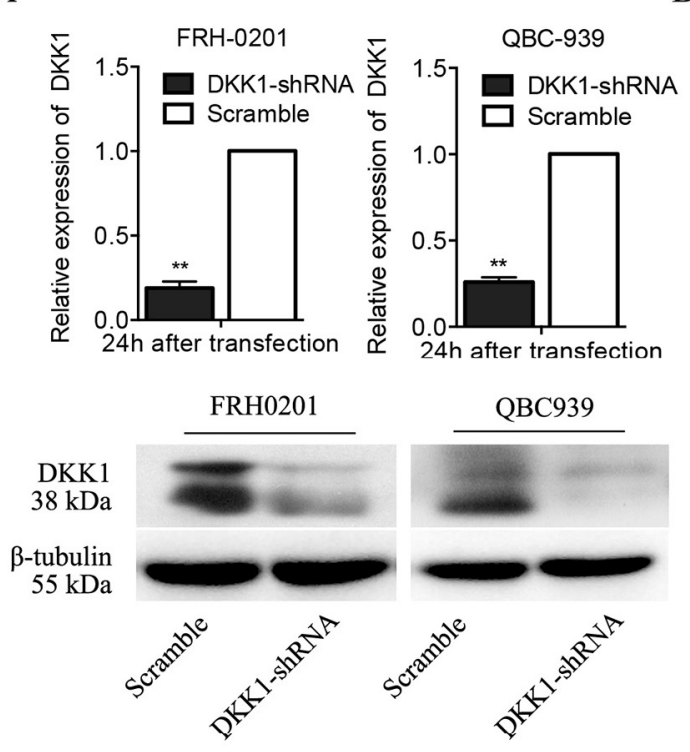

C

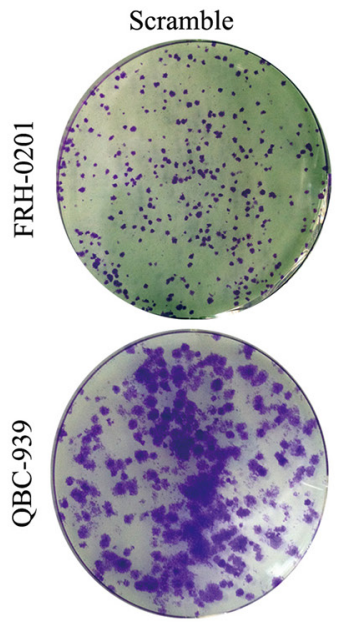

DKK1-shRNA
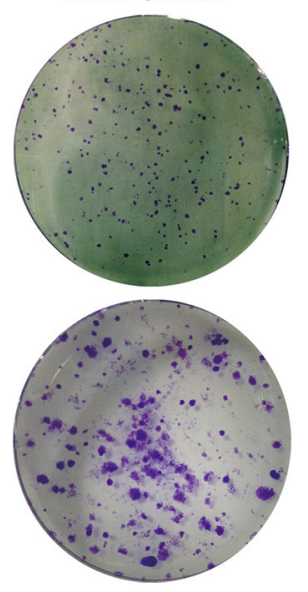

B

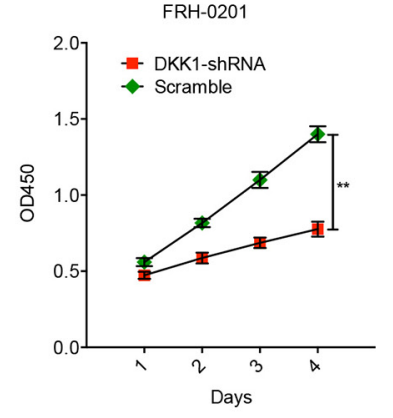

QBC-939
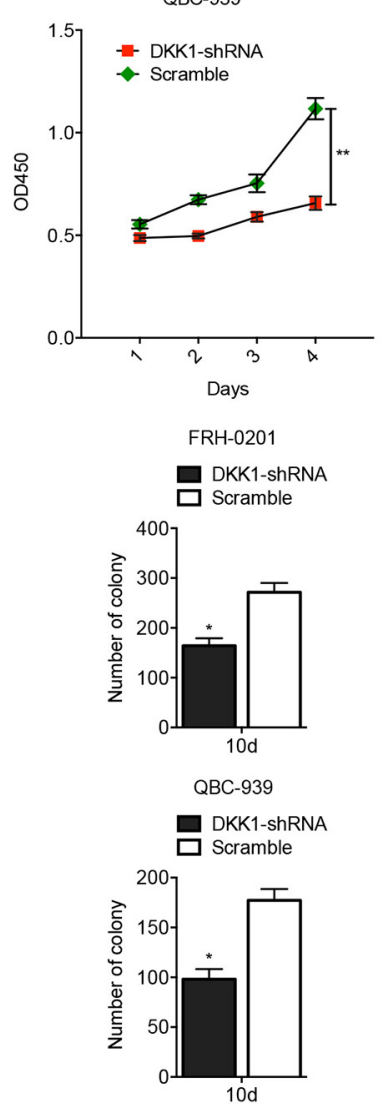

Figure 2: Transfection of DKK1-shRNA represses proliferation and colony formation of QBC939 and FRH0201 cells in vitro. Real time PCR analysis revealed that DKK1 mRNA in DKK1-shRNA QBC939 and FRH0201 cells was significantly downregulated A. Similar results were obtained by Western blotting analysis (A). Down-regulation of DKK1 expression by DKK1-shRNA significantly inhibited the proliferation of QBC939 and FRH0201 cells B. The number of colony was lower in DKK1-shRNA QBC939 and FRH0201 cells group than the control group $(\mathrm{C}) .{ }^{*} \mathrm{P}<0.05$. 
HCC cells [25]. In fact, the DKK1 promoter region contains several putative T-cell factor (TCF)-binding sites and was shown to be a direct target of activated $\beta$-catenin [33]. In addition, secreted DKK 1 could block its own transcription, thus creating a negative feedback loop [34]. In the present study, immunohistochemistry revealed that DKK1 levels were positively correlated with $\beta$-catenin expression in tissue samples from all thirty-seven HCCA cases. In addition, the expression of $\beta$-catenin and nuclear $\beta$-catenin were dramatically decreased in DKK1-shRNA QBC939 and FRH0201 cells. These findings indicate that $\beta$-catenin may be an important target downstream of DKK1.

MMP-7, also known as matrilysin, is a secreted protein implicated in the destruction of a broad range of extracellular matrix substrates in various cancers [35, 36]. MMP-7 is one of the most important target genes downstream of $\beta$-catenin signaling and plays a crucial role in promoting tumor cell migration and invasion [37, 38]. Recently, several reports have shown that MMP-
7 is up-regulated in both cholangiocarcinoma cells and tissues [39-41]. DKK1 promotes HCC cell migration and invasion through MMP-7 [21]. However, the relationship between DKK1 and MMP-7 in HCCA remains unclear. Herein, we demonstrate that MMP-7 is an important target downstream of DKK1 in HCCA cells. DKK1-shRNA markedly decreased $\beta$-catenin and MMP-7 protein levels in QBC939 and FRH0201 cells. Consistently, in human HCCA tissues, DKK1 level was positively correlated with $\beta$-catenin and MMP-7 expression, as well as tumor hilar lymphatic metastasis. Our findings suggest that DKK1 promotes HCCA cell migration and invasion, at least in part through promoting $\beta$-catenin/MMP-7 signaling.

In conclusion, our results indicate that DKK1 may be a crucial regulator in the progression of human HCCA, DKK1 exerts its pro-invasion function at least in part through the $\beta$-catenin/ MMP-7 signaling pathway, suggesting that DKK1 may be a potential target for HCCA therapy.
A

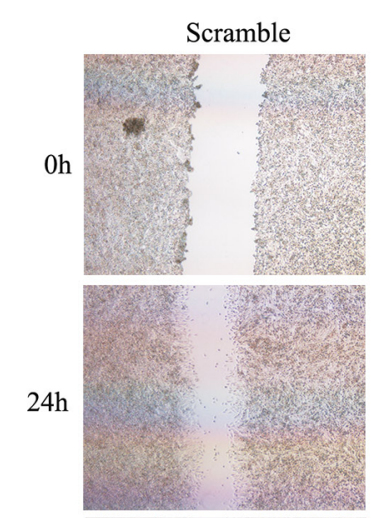

FRH-0201
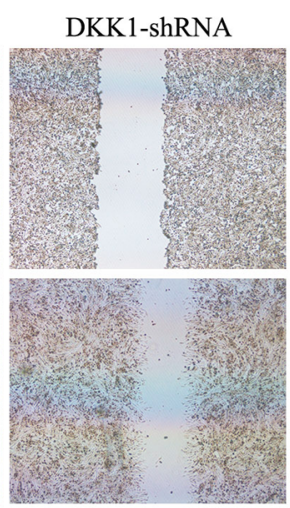

$48 \mathrm{~h}$
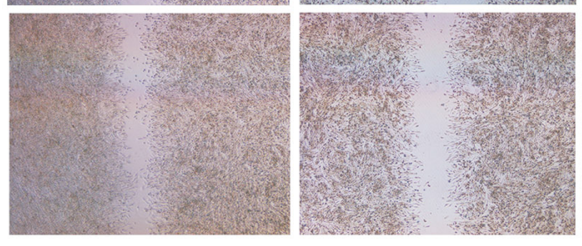

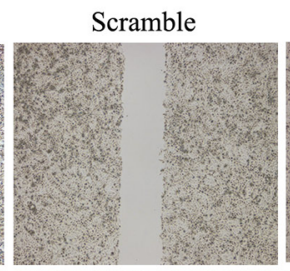

QBC-939

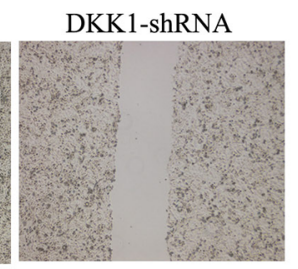

B

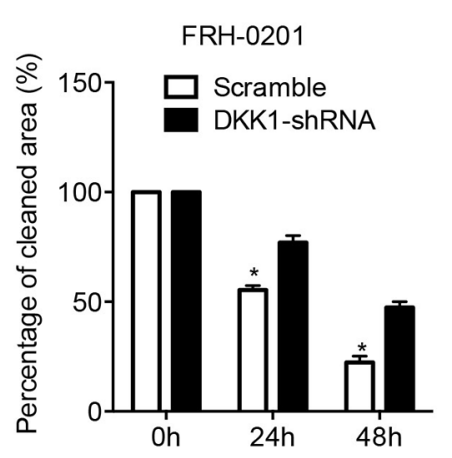

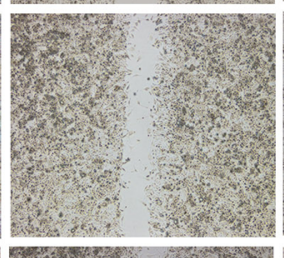

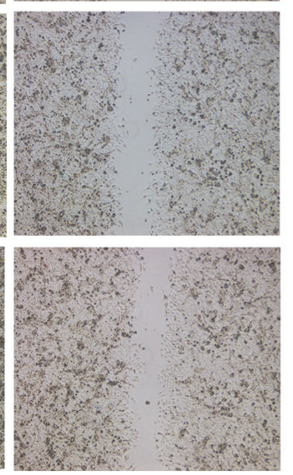

QBC-939

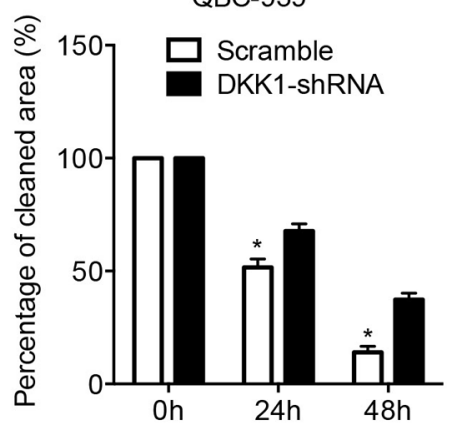

Figure 3: Transfection of DKK1-shRNA represses the migration of QBC939 and FRH0201 cells in vitro. In a wound healing assay, QBC939 and FRH0201 cells in the DKK1-shRNA group exhibited decreased migration ability compared with NC-shRNA group A. Cells were monitored every $24 \mathrm{~h}$ for 2 days to evaluate the rate of migration into the scratched area $\mathbf{B}$. ${ }^{*} \mathrm{P}<0.05$. 
A
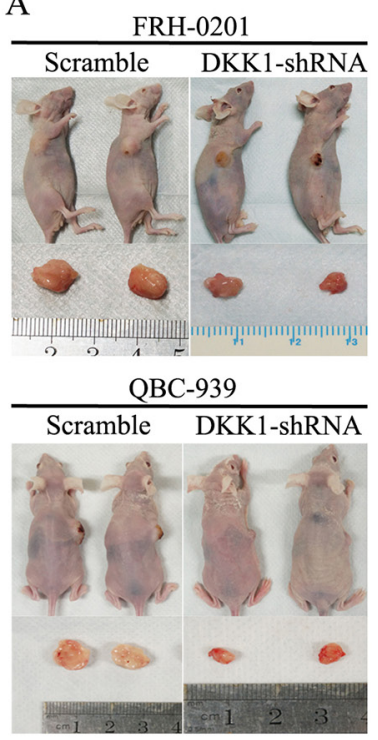

$\mathrm{C}$

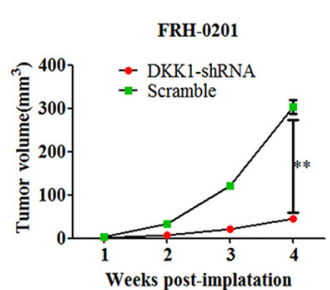

B
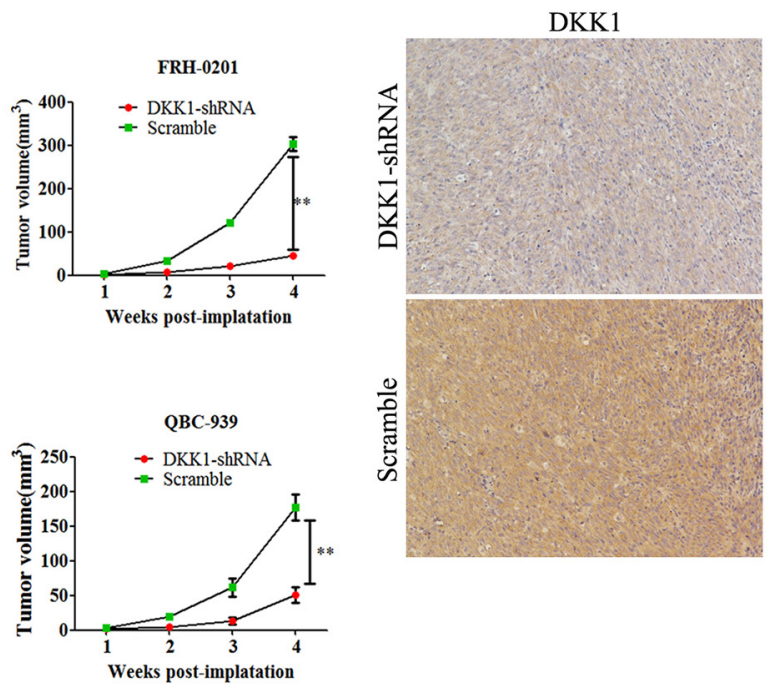

$\mathrm{D}$

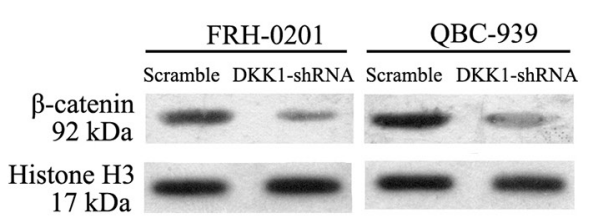

Figure 4: Transfection of DKK1-shRNA impaired QBC939 and FRH0201 cell tumor formation in vivo. Following downregulation of DKK1 expression, QBC939 and FRH0201 cells exhibited significantly diminished in vivo tumor formation ability compared with control cells. Control group QBC939 and FRH0201 cells generated large tumors on the left flanks of nude mice A, left panel. whereas smaller tumors were observed on the right flanks injected with DKK1-shRNA QBC939 and FRH0201 cells A, left panel. The tumor volume of the recipient mice is presented A, right panel. The expression of DKK1 was positively correlated with subcutaneous tumor volume B. Western blot revealed that shRNA-DKK1 remarkably decreased the levels of $\beta$-catenin $\mathbf{C}$. MMP7(C) and nuclear $\beta$-catenin D. protein levels of QBC939 and FRH0201 cells compared with control cells. ${ }^{*} \mathrm{P}<0.05$.

\section{MATERIALS AND METHODS}

\section{Patients and tissue specimens}

Tumor specimens were obtained from thirty-seven consecutive HCCA patients who underwent resection at the Department of Biliary-Pancreatic Surgery, Sun Yatsen Memorial Hospital. None of the patients received chemotherapy or radiation therapy prior to radical tumor resection. The difference and significance of DKK1 expression among those patients were investigated. The detailed clinicopathological characteristics of all patients are presented in Table 1. The project was approved by the ethics committee of the Sun Yat-sen Memorial Hospital. Written informed consent was obtained from either the patient or guardian.

\section{Immunohistochemistry}

Immunohistochemistry was performed according to the protocol provided with the PV Two-Step Kit instructions and as previously described [42]. Details regarding primary and secondary antibodies are provided in Supplementary Table S1. DKK1, $\beta$-catenin and MMP-7 expression was evaluated under a light microscope. The staining was independently evaluated by two investigators who were unaware of the clinical data. For each specimen, five images of representative areas were acquired, and tumor cells were counted. The percentages of positively stained cells were determined by examination under a microscope of 30 randomly selected foci, which were each composed of more than 100 cells. The intensity of DKK1 staining was evaluated as follows: strongly positive samples (scored as +++ ) had dark brown staining in $>50 \%$ of tumor cells, completely obscuring the cytoplasm; moderately positive (scored as ++ ) had dark brown staining in 25 to $50 \%$ of tumor cells obscuring the cytoplasm; weakly positive $(+)$ showed a lesser degree of brown staining in the tumor cell cytoplasm; and "absent" (scored as -) exhibited no appreciable staining in tumor cells. Strong and moderate scores were regarded as positive results $[22,25]$. 


\section{Cell culture}

Two human HCCA cell lines QBC939 and FRH0201 were obtained from the Cell Bank of the Chinese Academy of Sciences (Shanghai, China) and cultured in RPMI 1640 (Invitrogen Co., Carlsbad, CA) supplemented with $10 \%$ heat-inactivated fetal bovine serum (Invitrogen) as recommended by the supplier. All cultures were maintained in a humidified atmosphere containing 5\% $\mathrm{CO}_{2}$ at $37^{\circ} \mathrm{C}$.

\section{Virus production and transduction}

Lentiviral vectors expressing short hairpin RNA (shRNA) targeting human DKK1 (target sequence: shDKK1-1, 5-GATCCGTACCAAGCATAGGAGAAATT CAAGAGATTTCTCCTATGCTTGGTACTTTTTTG-3; sh-DKK1-2, 5-AATTCAAAAAAGTACCAAGCATAGG AGAAATCTCTTGAATTTCTCCTATGCTTGGTACG-3) and a negative control were purchased from GenePharma (Shanghai, China). All shRNA sequences were subjected to basic local alignment search tool (BLAST) search to confirm the absence of homology to any additional known coding sequences. QBC939 and FRH0201 cells were transfected with shRNA-DKK1. After puromycin selection, loss of DKK1 was confirmed by Quantitative RT-PCR (qRT-PCR) and Western blot analysis, and stable cell lines were established. A validated siRNA targeting DKK1 and the negative control were purchased from GenePharma (Shanghai, China). Transfection was performed as described previously [43].

\section{Quantitative RT-PCR and western blot analyses}

Knockdown of DKK1 in QBC939 and FRH0201cells was confirmed by real time polymerase chain reaction (qRT-PCR) as described [42]. Primer sets used for qRT-PCR amplification are presented in Supplementary Table S2. Western blot analyses were performed as described [42], protein lysates obtained from the cultured cells were subjected to sodiumdodecylsulfatepolyacrylamidegelelectrophoresis (SDS-PAGE) and were probed with primary antibodies recognizing DKK1 (1:2000), $\beta$-catenin (1:5000) and MMP-7(1:5000). After incubation with appropriate horseradish peroxidase (HRP)-conjugated secondary antibodies (Jackson Innumoresearch, USA), protein bands were visualized using enhanced chemiluminescence (ECL) plus Western blotting detection reagents followed by exposure to Hyper-films (Amersham, UK). Details regarding the primary and secondary antibodies were provided in Supplementary Table S1.

\section{Cell proliferation assay}

Cell proliferation assay was performed as previously described [42]. Briefly, HCCA cells were inoculated in 96-well plates at the density of $5 \times 10^{3} /$ well for 24 $\mathrm{h}$, and 3 repetitive wells were prepared for each group. After the corresponding treatments, the cells were treated with $10 \mu \mathrm{l}$ Cell Counting Kit-8 (CCK-8) (Dojindo Molecular Technologies) in an incubator for $2 \mathrm{~h}$. The absorbance at $450 \mathrm{~nm}$ was measured by an enzyme linked immunosorbent assay (ELISA) plate reader to calculate the cell growth inhibition rate (\%) according to the formulation: Inhibition rate $(\%)=\left(\mathrm{OD}_{\text {blank }}-\mathrm{OD}_{\text {experiment }}\right)$ $\mathrm{OD}_{\text {blank }} \times 100 \%$. The absorbance of the negative control (OD) was considered to be $0 \%$.

\section{Colony formation assay}

HCCA cells were plated in triplicate in six-well plates. After 7 days, the cells were rinsed with phosphatebuffered saline (PBS) twice, fixed with $10 \%$ formaldehyde, and stained with $0.1 \%$ crystal violet in $10 \%$ ethanol and the numbers of colonies were counted.

\section{Wound healing assay}

Briefly, $8 \times 10^{5}$ HCCA cells were cultured on a 35$\mathrm{mm}$ dish and incubated overnight. A wound was created by scratching with a $250-\mu l$ microtip, and the cells were washed once with complete medium to remove detached cells. An image of the original wound was captured under a microscope. Dishes were then returned to the incubator for $24 \mathrm{~h}$, and a second image of the wound was obtained. The width of the wound was measured with AxioVision LE software (Zeiss, Oberkochen, Germany). The difference between the widths is taken as the migration distance.

\section{In vivo subcutaneous xenografts}

All animal experimentation described in this study was performed in accordance with protocols approved by the Institutional Animal Care and Use Committee at Sun Yat-sen University. Briefly, $5 \times 10^{6}$ shRNA-DKK1 HCCA cells and control cells were suspended in $100 \mu \mathrm{l}$ PBS and were injected subcutaneously into six female nude mice (Balb/c nu/nu) (3-4 weeks old), respectively. Tumor volumes were monitored every 7 days by measuring the length and width with a caliper and using the formula $\left(\right.$ width $\left.^{2}\right) \times$ length $/ 2$. Mice were sacrificed 8 weeks after injection, and the tumors were isolated and measured.

\section{Statistics}

All experiments were performed at least in triplicate. Statistical analysis was conducted with the SPSS software package (version 13.0; SPSS, Chicago, IL). All experiments for cell cultures were carried out independently at least three times and in triplicate each time. All data were presented as mean \pm standard deviation (SD), we determined the significance of differences in the 
human HCCA data using Fisher's exact test, in the in vitro data using Student's t test, and in the in vivo data using the Mann-Whitney $U$ test. For all tests, a p-value less than 0.05 is considered to be statistically significant and indicated by asterisks in the figures. All p-values reported are the result of two-sided tests.

\section{ACKNOWLEDGMENTS}

This work was supported by The Special Research Foundation of the National Nature Science Foundation of China (81301865, 81300392 and 81302143), the Guangdong Science and Technology Foundation (2016A020215199). This work was supported by Grant [2013]163 from Key Laboratory of Malignant Tumor Molecular Mechanism and Translational Medicine of Guangzhou Bureau of Science and Information Technology.

\section{CONFLICTS OF INTEREST}

The authors declare no conflict of interest.

\section{REFERENCES}

1. de Groen PC, Gores GJ, LaRusso NF, Gunderson LL, Nagorney DM. Biliary tract cancers. N Engl J Med 1999, 341: 1368-1378.

2. Khan SA, Davidson BR, Goldin R, Pereira SP, Rosenberg WM, Taylor-Robinson SD, Thillainayagam AV, Thomas HC, Thursz MR, Wasan H. Guidelines for the diagnosis and treatment of cholangiocarcinoma: consensus document. Gut 2002, 51: I1-I9.

3. Patel T. Cholangiocarcinoma. Nat Clin Pract Gastroenterol Hepatol 2006, 3: 33-42.

4. DeOliveira ML, Cunningham SC, Cameron JL, Kamangar F, Winter JM, Lillemoe KD, Choti MA, Yeo CJ, Schulick RD. Cholangiocarcinoma: thirty-one-year experience with 564 patients at a single institution. Ann Surg 2007, 245: 755-762.

5. Ercolani G, Dazzi A, Giovinazzo F, Ruzzenente A, Bassi C, Guglielmi A, Scarpa A, D'Errico A, Pinna AD. Intrahepatic, peri-hilar and distal cholangiocarcinoma: Three different locations of the same tumor or three different tumors? Eur J Surg Oncol 2015, 41: 1162-1169.

6. Rea DJ, Munoz-Juarez M, Farnell MB, Donohue JH, Que FG, Crownhart B, Larson D, Nagorney DM. Major hepatic resection for hilar cholangiocarcinoma: analysis of 46 patients. Arch Surg 2004, 139: 514-523, 523-525.

7. Figueras J, Llado L, Valls C, Serrano T, Ramos E, Fabregat J, Rafecas A, Torras J, Jaurrieta E. Changing strategies in diagnosis and management of hilar cholangiocarcinoma. Liver Transpl 2000, 6: 786-794.

8. Khan SA, Thomas HC, Davidson BR, Taylor-Robinson SD. Cholangiocarcinoma. Lancet 2005, 366: 1303-1314.
9. Glinka A, Wu W, Delius H, Monaghan AP, Blumenstock C, Niehrs C. Dickkopf-1 is a member of a new family of secreted proteins and functions in head induction. Nature 1998, 391: 357-362.

10. Semenov MV, Tamai K, Brott BK, Kuhl M, Sokol S, He $\mathrm{X}$. Head inducer Dickkopf-1 is a ligand for Wnt coreceptor LRP6. Curr Biol 2001, 11: 951-961.

11. Cadigan KM, Liu YI. Wnt signaling: complexity at the surface. J Cell Sci 2006, 119: 395-402.

12. Mikheev AM, Mikheeva SA, Liu B, Cohen P, Zarbl H. A functional genomics approach for the identification of putative tumor suppressor genes: Dickkopf-1 as suppressor of HeLa cell transformation. Carcinogenesis 2004, 25: 47-59.

13. You L, He B, Uematsu K, Xu Z, Mazieres J, Lee A, McCormick F, Jablons DM. Inhibition of Wnt-1 signaling induces apoptosis in beta-catenin-deficient mesothelioma cells. Cancer Res 2004, 64: 3474-3478.

14. Forget MA, Turcotte S, Beauseigle D, Godin-Ethier J, Pelletier S, Martin J, Tanguay S, Lapointe R. The Wnt pathway regulator DKK1 is preferentially expressed in hormone-resistant breast tumours and in some common cancer types. Br J Cancer 2007, 96: 646-653.

15. Yamabuki T, Takano A, Hayama S, Ishikawa N, Kato T, Miyamoto M, Ito T, Ito H, Miyagi Y, Nakayama H, Fujita M, Hosokawa M, Tsuchiya E, et al. Dikkopf-1 as a novel serologic and prognostic biomarker for lung and esophageal carcinomas. Cancer Res 2007, 67: 2517-2525.

16. Darlavoix T, Seelentag W, Yan P, Bachmann A, Bosman FT. Altered expression of CD44 and DKK1 in the progression of Barrett's esophagus to esophageal adenocarcinoma. Virchows Arch 2009, 454: 629-637.

17. Makino T, Yamasaki M, Takemasa I, Takeno A, Nakamura Y, Miyata H, Takiguchi S, Fujiwara Y, Matsuura N, Mori M, Doki Y. Dickkopf-1 expression as a marker for predicting clinical outcome in esophageal squamous cell carcinoma. Ann Surg Oncol 2009, 16: 2058-2064.

18. Patil MA, Chua MS, Pan KH, Lin R, Lih CJ, Cheung ST, Ho C, Li R, Fan ST, Cohen SN, Chen X, So S. An integrated data analysis approach to characterize genes highly expressed in hepatocellular carcinoma. Oncogene 2005, 24: 3737-3747.

19. Li S, Qin X, Guo X, Cui A, He Y, Wei S, Wang X, Shan B. Dickkopf-1 is oncogenic and involved in invasive growth in non small cell lung cancer. PLoS One 2013, 8: e84944.

20. Takahashi N, Fukushima T, Yorita K, Tanaka H, Chijiiwa $\mathrm{K}$, Kataoka H. Dickkopf-1 is overexpressed in human pancreatic ductal adenocarcinoma cells and is involved in invasive growth. Int J Cancer 2010, 126: 1611-1620.

21. Chen L, Li M, Li Q, Wang CJ, Xie SQ. DKK1 promotes hepatocellular carcinoma cell migration and invasion through beta-catenin/MMP7 signaling pathway. Mol Cancer 2013, 12: 157.

22. Shi RY, Yang XR, Shen QJ, Yang LX, Xu Y, Qiu SJ, Sun YF, Zhang X, Wang Z, Zhu K, Qin WX, Tang ZY, Fan $\mathrm{J}$, et al. High expression of Dickkopf-related protein 1 
is related to lymphatic metastasis and indicates poor prognosis in intrahepatic cholangiocarcinoma patients after surgery. Cancer 2013, 119: 993-1003.

23. Fingas CD, Blechacz BR, Smoot RL, Guicciardi ME, Mott J, Bronk SF, Werneburg NW, Sirica AE, Gores GJ. A smac mimetic reduces TNF related apoptosis inducing ligand (TRAIL)-induced invasion and metastasis of cholangiocarcinoma cells. Hepatology 2010, 52: 550-561.

24. Klaus A, Birchmeier W. Wnt signalling and its impact on development and cancer. Nat Rev Cancer 2008, 8: 387-398.

25. Yu B, Yang X, Xu Y, Yao G, Shu H, Lin B, Hood L, Wang H, Yang S, Gu J, Fan J, Qin W. Elevated expression of DKK1 is associated with cytoplasmic/nuclear betacatenin accumulation and poor prognosis in hepatocellular carcinomas. J Hepatol 2009, 50: 948-957.

26. Lamb R, Ablett MP, Spence K, Landberg G, Sims AH, Clarke RB. Wnt pathway activity in breast cancer sub-types and stem-like cells. PLoS One 2013, 8: e67811.

27. Rachner TD, Gobel A, Benad-Mehner P, Hofbauer LC, Rauner M. Dickkopf-1 as a mediator and novel target in malignant bone disease. Cancer Lett 2014, 346: 172-177.

28. Nusse R. Wnt signaling in disease and in development. Cell Res 2005, 15: 28-32.

29. Menezes ME, Devine DJ, Shevde LA, Samant RS. Dickkopf1: a tumor suppressor or metastasis promoter? Int J Cancer 2012, 130: 1477-1483.

30. Hu P, Chu J, Wu Y, Sun L, Lv X, Zhu Y, Li J, Guo Q, Gong C, Liu B, Su S. NBAT1 suppresses breast cancer metastasis by regulating DKK1 via PRC2. Oncotarget 2015, 6: 3241032425. doi: 10.18632/oncotarget.5609.

31. Cho SW, Lee EJ, Kim H, Kim SH, Ahn HY, Kim YA, Yi KH, Park DJ, Shin CS, Ahn SH, Cho BY, Park YJ. Dickkopf-1 inhibits thyroid cancer cell survival and migration through regulation of beta-catenin/E-cadherin signaling. Mol Cell Endocrinol 2013, 366: 90-98.

32. He X, Li S, Luo X, Hu D, Cai T, Huang K, Zhou W, Chen J. Expression of DKK1 and beta-catenin in epidermal neoplasms and their correlation. Int J Clin Exp Med 2015, 8: 18843-18848.

33. Niida A, Hiroko T, Kasai M, Furukawa Y, Nakamura Y, Suzuki Y, Sugano S, Akiyama T. DKK1, a negative regulator of Wnt signaling, is a target of the beta-catenin/ TCF pathway. Oncogene 2004, 23: 8520-8526.

34. Gonzalez-Sancho JM, Aguilera O, Garcia JM, PendasFranco N, Pena C, Cal S, Garcia DHA, Bonilla F, Munoz A. The Wnt antagonist DICKKOPF-1 gene is a downstream target of beta-catenin/TCF and is downregulated in human colon cancer. Oncogene 2005, 24: 1098-1103.
35. Basu S, Thorat R, Dalal SN. MMP7 is required to mediate cell invasion and tumor formation upon Plakophilin3 loss. PLoS One 2015, 10: e123979.

36. Kesh K, Subramanian L, Ghosh N, Gupta V, Gupta A, Bhattacharya S, Mahapatra NR, Swarnakar S. Association of MMP7 -181A->G Promoter Polymorphism with Gastric Cancer Risk: INFLUENCE OF NICOTINE IN DIFFERENTIAL ALLELE-SPECIFIC TRANSCRIPTION VIA INCREASED PHOSPHORYLATION OF cAMPRESPONSE ELEMENT-BINDING PROTEIN (CREB). J Biol Chem 2015, 290: 14391-14406.

37. Nakachi S, Nakazato T, Ishikawa C, Kimura R, Mann DA, Senba M, Masuzaki H, Mori N. Human T-cell leukemia virus type 1 tax transactivates the matrix metalloproteinase 7 gene via JunD/AP-1 signaling. Biochim Biophys Acta 2011, 1813: 731-741.

38. Oshima T, Akaike M, Yoshihara K, Shiozawa M, Yamamoto N, Sato T, Yamada R, Fujii S, Rino Y, Kunisaki C, Tanaka $\mathrm{K}$, Masuda M, Imada T. Clinicopathological significance of the gene expression of matrix metalloproteinase-7, insulin-like growth factor-1, insulin-like growth factor-2 and insulin-like growth factor-1 receptor in patients with colorectal cancer: insulin-like growth factor-1 receptor gene expression is a useful predictor of liver metastasis from colorectal cancer. Oncol Rep 2008, 20: 359-364.

39. Leelawat K, Narong S, Wannaprasert J, Ratanashu-ek T. Prospective study of MMP7 serum levels in the diagnosis of cholangiocarcinoma. World J Gastroenterol 2010, 16: 4697-4703.

40. Lumachi F, Lo RG, Tozzoli R, D'Aurizio F, Facomer F, Chiara GB, Basso SM. Measurement of serum carcinoembryonic antigen, carbohydrate antigen 19-9, cytokeratin-19 fragment and matrix metalloproteinase-7 for detecting cholangiocarcinoma: a preliminary case-control study. Anticancer Res 2014, 34: 6663-6667.

41. Jo HJ, Shim HE, Han ME, Kim HJ, Kim KS, Baek S, Choi KU, Hur GY, Oh SO. WTAP regulates migration and invasion of cholangiocarcinoma cells. J Gastroenterol 2013, 48: 1271-1282.

42. Zhang R, Wu WR, Shi XD, Xu LB, Zhu MS, Zeng H, Liu C. Dysregulation of Bmil promotes malignant transformation of hepatic progenitor cells. Oncogenesis 2016, 5: e203.

43. Wu WR, Sun H, Zhang R, Yu XH, Shi XD, Zhu MS, Zeng H, Yan LX, Xu LB, Liu C. Methylation-associated silencing of miR-200b facilitates human hepatocellular carcinoma progression by directly targeting BMI1. Oncotarget 2016, 7: 18684-18693. doi: 10.18632/oncotarget.7629. 